\title{
Improvement of absolute pitch naming
}

MICHAEL TERMAN

COLUMBIA UNIVERSITY ${ }^{1}$

\begin{abstract}
Abstraet
Ss improved their accuracy in naming sine tones in an "absolute context" in which an intertrial distractive procedure was used to reduce "relative" cues. This result does not support the popular assumption that absolute pitch is an innate and inviolable "gift."
\end{abstract}

\section{Introduction}

Absolute pitch (AP) has been casually defined as the ability to name or produce a pitch without reference to any other pitch. Revesz (1954) has suggested that people with this ability judge pitch on the basis of "tone color" rather than "tone height." This subjective distinction defies behavioral analysis since it evades operational definition. It would thus seem necessary to define AP by experimental procedure, so that stimulus, response and intertrial variables are throughly specified.

Lundin \& Allen (1962) trained Ss to identify piano tones presented $20 \mathrm{sec}$. apart (with intertrial silence) in the two octave range above middle $\mathrm{C}$. Their procedure was refined in the present experiment, for it was noted that stimulus tones may be recalled and reproduced with high accuracy after 20 sec. of intertrial silence, and thus used as relative cues, weakening the "absolute" construct.

\section{Method}

Seven magnetic tapes were programmed for use with TMI-Grolier Min/Max II teaching machines which were modified so that a three step response-answer-correction sequence could be followed on each training trial. Sine tone stimuli were used to reduce the complex cues associated with musical tones. Each tape contained 25 randomized trials, comprising the two octave range of 25 equal-tempered pitches surrounding middle C ( $A=440.0 \mathrm{cps})$. Ss used a musical nomenclature which referred to pitch name and octave height. Individual tapes were not presented consecutively, but were repeated three to five times in the course of the experiment. The procedure for one training trial, constituting an operational definition of absolute pitch naming, is described in Table 1. A distractive procedure consisting of silence, random sweeps of loud and soft sine tones (similar to a fire-siren pattern), and vocalization by the $S$ was employed to minimize intertrial cues.

Eleven adult Ss, who had various musical backgrounds but did not claim to "have" AP, were run in weekly 1-hr. sessions, two training tapes per session.

\section{Results and Diseussion}

In order to evaluate the judgments, criteria for "correct" and "error" responses were specified. As emphasized by Graham \& Ratoosh (1962), the defined response continuum (here, the musical scale) need not reflect the physical dimensions of the stimulus continuum. Occurrence of pitch names as a function of individual stimulus frequencies did vary widely. Sample distributions are shown in Fig. 1, with appropriate pitch names indicated in quotes. Since the distributions were all modal on the defined stimulus frequencies, and tended to decrease monotonically with increasing distance, accuracy scores were based on the deviation of pitch names in musical halfsteps from the defined stimulus frequencies. (The distance between neighboring tones on the stimulus continuum is defined by music theory as a halfstep.) A general indication of group accuracy may be seen in Fig. 1, where the modal pitch name for each stimulus frequency is plotted (solid line). Pitch naming accuracy was highest in the extremes of the stimulus continuum, and a roughly U-shaped function connects the extremes, suggesting that relative accuracy for naming the various stimuli was partially a function of a procedural artifact-the limited range of test frequencies.

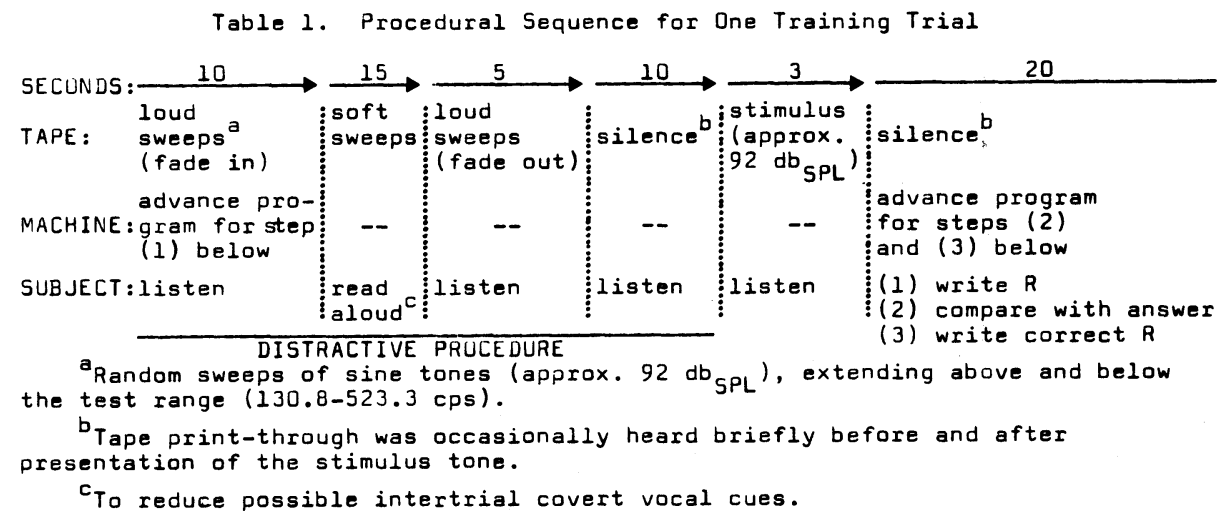

Psychon.. Sci., 1965, Vol. 3 


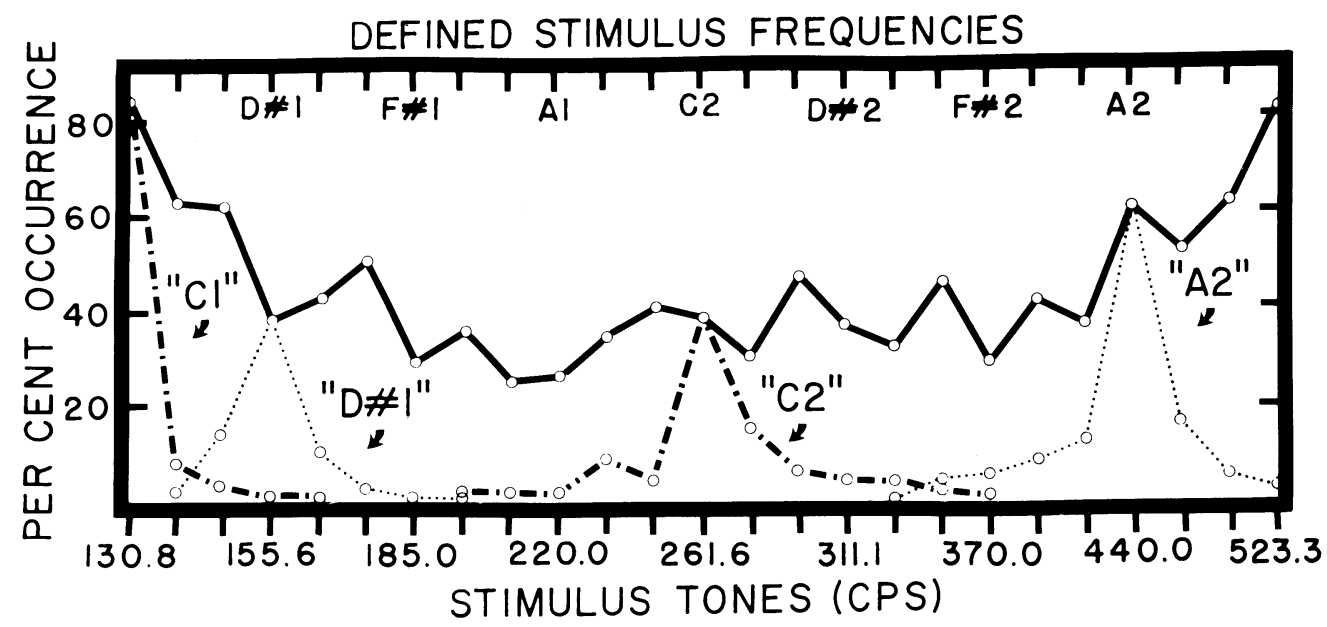

Fig. 1. Per cent occurrence of pitch names as a function of stimulus frequency presented. Group data based on the last eight training tapes. Solid line connects modal pitch name for each stimulus frequency.

Sample individual learning curves are shown in Fig. 2, based on four score levels with the following criteria for "correct" responses: responses naming only the defined stimulus frequency (A); and responses occurring within one (B), two (C) or three (D) halfsteps of the defined stimulus frequency, plus octave errors. Octave errors rarely occurred after the first few training sessions, but were included in scores B-D because of Révész's (1954) report that they are common for people who "have" AP.

Accuracy scores, as well as rates of score increment, show great differences for each S. For example, both SF and JM began training with score A levels of approximately 10-15\% correct, but at the conclusion of training JM scored $38 \%$ and SF scored $75 \%$, much closer to the final score of SS $(82 \%)$, who was the

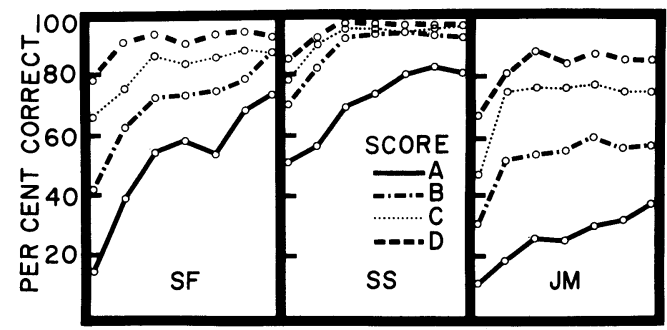

TRAINING TAPES (BLOCKS OF 4)

Fig. 2. Mean per cent of correct responses for successive blocks of four training tapes. Score criteria A-D described in text. overall high-scorer. Ten out of 11 Ss did show some score improvement. There was no clear relation between the extent of improvement and S's musical experience. Comparison of score levels A-D indicates that the generalization of pitch names was most often close to the defined stimulus frequency. All Ss achieved scores of $84 \%$ correct or better on the level of score D. In some cases a steady level of performance was reached in scores $\mathrm{C}$ or $\mathrm{D}$ while scores $\mathrm{A}$ and $\mathrm{B}$ continued to rise, but extended training would have been necessary to ascertain that asymptotes were, in fact, achieved.

On the anecdotal level, at least, the distractive procedure was found to be very effective in separating trials. Only SS reported remembering stimulus tones through the distractive procedure and using them as relative cues.

\section{References}

Graham, C. H., \& Ratoosh, P. Notes on some interrelations of sensory psychology, perception, and behavior. In S. Koch (Ed), Psychology a study of a science. Vol. 4. New York: McGrawHill, 1962. Pp. 483-514.

Lundin, R. W., \& Allen, J. D. A technique for training perfect pitch. Psychol. Rec., 1962, 12, 139-146.

Révész, G. Introduction to the psychology of music. Norman: University of Oklahoma Press, 1954.

\section{Note}

1. Now at Brown University. I thank H. S. Terrace and D. F , Johnson for their guidance, J. W. Kling for his critical reading of the manuscript, and C. M. Harris and V. Ussachevsky for laboratory facilities. 